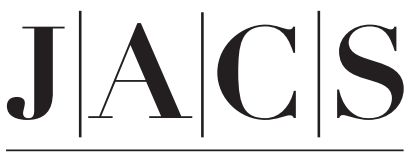

A R T I C L E S

Published on Web 05/12/2004

\title{
Electron Transport in Two-Dimensional Arrays of Gold Nanocrystals Investigated by Scanning Electrochemical Microscopy
}

\author{
Peter Liljeroth, ${ }^{\dagger}$ Daniël Vanmaekelbergh, ${ }^{\dagger}$ Virginia Ruiz, ${ }^{\ddagger}$ Kyösti Kontturi, ${ }^{\ddagger}$ \\ Hua Jiang,,$\| l$ Esko Kauppinen," and Bernadette M. Quinn ${ }^{*}, \neq$ \\ Contribution from the Laboratory of Physical Chemistry and Electrochemistry, \\ Helsinki University of Technology, P.O. Box 6100, FIN-02015 HUT, Finland, Condensed Matter \\ and Interfaces, Debye Institute, University of Utrecht, P.O. Box 80000, 3508 TA Utrecht, \\ The Netherlands, VTT Processes, Aerosol Technology Group, P.O. Box 1602, FIN-02044 VTT, \\ Espoo, Finland, and Center for New Materials, Helsinki University of Technology, \\ P.O. Box 1602 FIN-02044 VTT, Espoo, Finland
}

Received February 6, 2004; E-mail: bquinn@cc.hut.fi

\begin{abstract}
This article reports the use of the scanning electrochemical microscope (SECM) to investigate the electronic properties of Langmuir monolayers of alkane thiol protected gold nanocrystals (NCs). A substantial increase in monolayer conductivity upon mechanical compression of the Au NC monolayer is reported for the first time. This may be the room temperature signature of the insulator to metal transition previously reported for comparable silver NC monolayers. Factors influencing the conductivity of the monolayer NC array are discussed.
\end{abstract}

\section{Introduction}

Nanometer-sized metals and semiconductors are the focus of intense research as potential building blocks for future nanoscale devices. ${ }^{1}$ Recent progress in the liquid-phase preparation of narrow size distribution metal and semiconductor nanocrystals (NCs) has resulted in a considerable increase both in our fundamental understanding of their size-dependent optical and electronic properties and in the number of applications utilizing NCs. ${ }^{1 \mathrm{~b}, \mathrm{e}, 2}$

Metal nanocrystals passivated by an organic monolayer (also known as monolayer-protected clusters ${ }^{1 \mathrm{~b}}$ ) exhibit size-dependent charge storage properties when dispersed in solution. ${ }^{2 \mathrm{a}, 3}$ The collective properties of their assemblies can be tuned by

\footnotetext{
$\dagger$ University of Utrecht.

$\doteqdot$ Laboratory of Physical Chemistry and Electrochemistry, Helsinki University of Technology.

$\S$ VTT Processes.

"Center for New Materials, Helsinki University of Technology.

(1) (a) Markovich, G.; Collier, C. P.; Henrichs, S. E.; Remacle, F.; Levine, R. D.; Heath, J. R. Acc. Chem. Res. 1999, 32, 415. (b) Templeton, A. C.; Wuelfing, W. P.; Murray, R. W. Acc. Chem. Res. 2000, 33, 27. (c) Collier, C. P.; Vossmeyer, T.; Heath, J. R. Annu. Rev. Phys. Chem. 1998, 49, 371. (d) Shenhar, R.; Rotello, V. M. Acc. Chem. Res. 2003, 36, 549. (e) Brust, M.; Walker, M.; Bethell, D.; Schiffrin, D. J.; Whyman, R. J. Chem. Soc., Chem. Commun. 1994, 801. (f) Roest, A. L.; Kelly, J. J.; Vanmaekelbergh, D.; Meulenkamp, E. A. Phys. Rev. Lett. 2002, 89, 036801. (g) Shim, M.; Guyot-Sionnest, P. Nature 2000, 407, 981. (h) Yu, D.; Wang, C.; GuyotSionnest, P. Science 2003, 300, 1277.

(2) (a) Chen, S.; Ingram, R. S.; Hostetler, M. J.; Pietron, J. J.; Murray, R. W.; Schaaff, T. G.; Khoury, J. T.; Alvarez, M. M.; Whetten, R. L. Science 1998, 280, 2098. (b) Collier, C. P.; Saykally, R. J.; Shiang, J. J.; Henrichs, S. E.; Heath, J. R. Science 1997, 277, 1978. (c) Murray, C. B.; Kagan, C. R.; Bawendi, M. G. Annu. Rev. Mater. Sci. 2000, 30, 545. (d) Murray, C. B.; Norris, D. J.; Bawendi, M. G. J. Am. Chem. Soc. 1993, 115, 8706. (e) Klimov, V. I.; Mikhailovsky, A. A.; Xu, S.; Malko, A.; Hollingsworth, J. A.; Leatherdale, C. A.; Eisler, H. J.; Bawendi, M. G. Science 2000, 290, 314. (f) Taton, T. A.; Mirkin, C. A.; Letsinger, R. L. Science 2000, 289, 1757.
}

variation of the core size, shape, and interparticle coupling. ${ }^{2 \mathrm{~b}, 4}$ Pioneering work by Heath and co-workers demonstrated that it was possible to reversibly tune a Langmuir monolayer of thiolpassivated silver nanocrystals through an insulator-metal (IM) transition simply by mechanical compression of the monolayer. $^{2 b}$ At large interparticle distances (in the weak coupling regime), the superlattice is a Mott insulator with a Coulomb band gap described by the charging energies of the individual NC lattice sites. Upon monolayer compression, the interparticle separation is decreased, and the monolayer undergoes a sharp transition to the metallic state (characterized by a positive temperature coefficient of resistance) when the exchange coupling energy between adjacent NCs exceeds the charging energy, the so-called Mott-Hubbard transition. ${ }^{\text {la }}$ This reversible I-M transition was experimentally verified initially by impedance, optical, and tunneling spectroscopies. ${ }^{2 b, 5}$ The observed transition was noted to be dependent on the NC core size (charging energy), length of the thiol chain (interparticle separation), temperature $(k T)$, and the size/shape distribution (disorder) of the NCs making up the lattice. ${ }^{1 \mathrm{a}, 4 \mathrm{~g}}$ Temperature-

(3) Pietron, J. J.; Hicks, J. F.; Murray, R. W. J. Am. Chem. Soc. 1999, 121, 5565 .

(4) (a) Wuelfing, W. P.; Murray, R. W. J. Phys. Chem. B 2002, 106, 3139. (b) Hicks, J. F.; Zamborini, F. P.; Osisek, A. J.; Murray, R. W. J. Am. Chem Soc. 2001, 123, 7048. (c) Zamborini, F. P.; Hicks, J. F.; Murray, R. W. J. Am. Chem. Soc. 2000, 122, 4514. (d) Wuelfing, W. P.; Green, S. J.; Pietron J. J.; Cliffel, D. E.; Murray, R. W. J. Am. Chem. Soc. 2000, 122, 11465. (e) Chen, S.; Murray, R. W. J. Phys. Chem. B 1999, 103, 9996. (f) Remacle, F.; Beverly, K. C.; Heath, J. R.; Levine, R. D. J. Phys. Chem. B 2002 106, 4116. (g) Beverly, K. C.; Sampaio, J. F.; Heath, J. R. J. Phys. Chem. $B$ 2002, 106, 2131. (h) Sampaio, J. F.; Beverly, K. C.; Heath, J. R. J. Phys Chem. B 2001, 105, 8797 .

(5) (a) Kim, S. H.; Medeiros-Ribeiro, G.; Ohlberg, D. A. A.; Williams, R. S Heath, J. R. J. Phys. Chem. B 1999, 103, 10341. (b) Markovich, G.; Collier, C. P.; Heath, J. R. Phys. Rev. Lett. 1998, 80, 3807. (c) Remacle, F.; Collier, C. P.; Heath, J. R.; Levine, R. D. Chem. Phys. Lett. 1998, 291, 453. 
dependent transport measurements, however, revealed nonmetallic film behavior at decreased temperatures for films that had been compressed above the $\mathrm{I}-\mathrm{M}$ transition. ${ }^{4 \mathrm{~g}, \mathrm{~h}}$ Extensive theoretical work by Remacle, Levine, and co-workers on the role of disorder on the $\mathrm{I}-\mathrm{M}$ transition revealed that disorder due to the finite size and shape distribution of the NCs making up the lattice can smear out the transition and/or give a disorder driven Mott insulator-Anderson insulator transition. ${ }^{4 \mathrm{~g}, 6}$ Disorder within the superlattice tends to localize the states at the Fermi energy and makes it difficult to observe a true Mott-Hubbard I-M transition when probed at the mesoscopic length scale. A true $\mathrm{I}-\mathrm{M}$ transition is predicted to be experimentally attainable at very narrow $\mathrm{NC}$ size distributions ( $<3 \%$ polydispersity). ${ }^{4 \mathrm{~g}, 6 \mathrm{a}}$ Despite the chemical stability of gold nanocrystals and their well-documented self-assembly in NC monolayers, ${ }^{1 e, 7}$ to date a significant increase in monolayer conductivity upon compression has only been reported for silver NCs. This has been ascribed to the difficulty in preparing gold NCs with narrow size distributions. ${ }^{1 \mathrm{a}, 8}$ There is a recent report where the lateral conductivity of Au MPC monolayer was reported to increase exponentially with decreased interparticle separation. ${ }^{9}$ However, charging features apparent in current-voltage curves presented provide clear evidence of a Coulomb gap between individual NCs characteristic of an insulating NC monolayer. ${ }^{10}$

Here, we report the first observation of an exponential increase in monolayer conductivity upon compression of an $\mathrm{Au}$ $\mathrm{NC}$ monolayer, and the conductivity values presented are comparable to those previously reported for compressed $\mathrm{Ag}$ films above the I-M transition. ${ }^{4 \mathrm{~g}, \mathrm{~h}, 11}$ Scanning electrochemical microscopy (SECM), ${ }^{12}$ an advanced electrochemical technique, is used to follow the lateral conductivity of a series of $\mathrm{Au} \mathrm{NC}$ Langmuir monolayers at increasing monolayer compression. Previously, this technique has been used to probe lateral proton and amphiphile diffusion in Langmuir monolayers, ${ }^{13}$ the $\mathrm{I}-\mathrm{M}$ transition in an Ag nanoparticle monolayer, ${ }^{14}$ and local injection and lateral propagation of charge in ultrathin polymer and metal nanoparticle films. ${ }^{15}$ Essentially, SECM involves electrolysis of a solution redox mediator at a microelectrode (SECM tip), and monitoring the resulting tip current as a function of the tip-NC monolayer distance. This local depletion of the redox mediator (process (1) in Scheme 1) sets up an electrochemical potential gradient in the $\mathrm{NC}$ monolayer that causes electron transport to take place (process (2) in Scheme 1). The electrons

(6) (a) Beverly, K. C.; Sample, J. L.; Sampaio, J. F.; Remacle, F.; Heath, J. R.; Levine, R. D. Proc. Natl. Acad. Sci. U.S.A. 2002, 99, 6456. (b) Remacle, F.; Levine, R. D. J. Phys. Chem. B 2001, 105, 2153.

(7) (a) Parthasarathy, R.; Lin, X.-M.; Jaeger, H. M. Phys. Rev. Lett. 2001, 87 186807. (b) Lin, X.-M.; Jaeger, H. M.; Sorensen, C. M.; Klabunde, K. J. J. Phys. Chem. B 2001, 105, 3353. (c) Kiely, C. J.; Fink, J.; Brust, M.; Bethell, D.; Schiffrin, D. J. Nature 1998, 396, 444. (d) Santhanam, V.; Liu, J.; Agarwal, R.; Andres, R. P. Langmuir 2003, 19, 7881.

(8) Lee, W.-Y.; Hostetler, M. J.; Murray, R. W.; Majda, M. Isr. J. Chem. 1997, 37,213

(9) Chen, S. Anal. Chim. Acta 2003, 496, 29.

(10) Yang, Y.; Pradhan, S.; Chen, S. J. Am. Chem. Soc. 2004, 126, 76.

(11) Beverly, K. C.; Heath, J. R. Personal communication, California Institute of Technology, Pasadena, CA 91125, 2004.

(12) Bard, A. J.; Faulkner, L. R. Electrochemical Methods, Fundamentals and Applications, 2nd ed.; John Wiley \& Sons: New York, 2001.

(13) (a) Slevin, C. J.; Unwin, P. R. J. Am. Chem. Soc. 2000, 122, 2597. (b) Zhang, J.; Unwin, P. R. J. Am. Chem. Soc. 2002, 124, 2379. (c) Zhang, J.; Slevin, C. J.; Morton, C.; Scott, P.; Walton, D. J.; Unwin, P. R. J. Phys. Chem. B 2001, 105, 11120 .

(14) Quinn, B. M.; Prieto, I.; Haram, S. K.; Bard, A. J. J. Phys. Chem. B 2001 105,7474 .

(15) (a) Mandler, D.; Unwin, P. R. J. Phys. Chem. B 2003, 107, 407. (b) Liljeroth P.; Quinn, B. M.; Ruiz, V.; Kontturi, K. Chem. Commun. 2003, 1570. (c) Ruiz, V.; Liljeroth, P.; Quinn, B. M.; Kontturi, K. Nano Lett. 2003, 3, 1459 .
Scheme 1. Schematic of the Experimental Arrangement Which Depicts the Mechanism of the SECM Response ${ }^{a}$

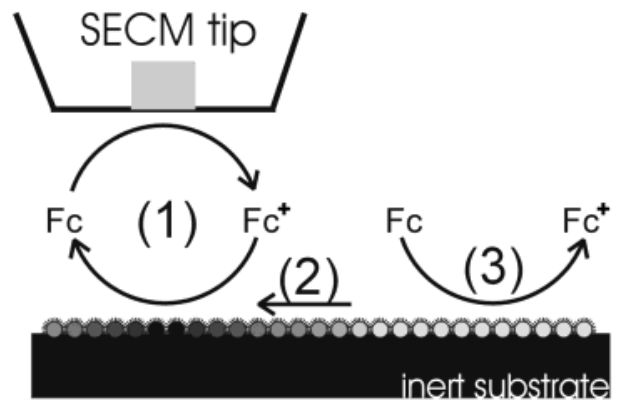

a (1) Depletion of the reduced form of ferrocenemethanol by the SECM tip, setting up an electrochemical potential gradient that drives lateral electron transport in the NC film (2). The electrons are supplied by the reverse reaction between the NCs and the solution redox couple outside the tip-substrate gap (3).

are injected into the film by the same redox couple outside the tip-substrate gap (process (3) in Scheme 1). Using a suitable model for the corresponding transport problem, we found that experimental approach curves yield quantitative information on the electron transport process in the NC monolayer. As SECM probes electron transport in a limited region of the NC film (few tens of micrometers), the stringent requirements of lateral film uniformity over the entire device and good electrode contacts in typical dc transport measurements can be relaxed. Other advantages of this technique compared with more conventional electroanalytical methods are that it is noninvasive and does not require the film to be externally biased. Also, as the film is assembled on an inert substrate, the measured response is due solely to charge injection and lateral charge transport in the film. Values for the conductivity of NC monolayers at different interparticle separations transferred by the Langmuir-Schaefer method to glass slides were determined using SECM. Recent literature methods to prepare NCs with improved monodispersity were used. The core size and the length of the thiol coat were noted to have a profound influence on the monolayer conductivity.

\section{Experimental Section}

Chemicals. Hexanethiol and dodecanethiol (Sigma) were used as received. All other chemicals were of the highest commercially available purity. Aqueous solutions were prepared using MQ-treated water (Millipore).

Preparation and Characterization of NCs. Hexanethiolate- and dodecanethiolate-protected gold particles (C6S-AuI and C12S-AuI) were synthesized according to a literature procedure proven to yield NCs with small core diameter. ${ }^{\text {le, }, 3,16}$ The size and polydispersity were quantified by differential pulse voltammetry (DPV) of solution-dispersed NCs. The appearance of well-resolved, regularly spaced current peaks in the DPV response due to quantized double layer charging (QDL) of the metal core was indicative of a highly monodisperse $(<10 \%)$ core size. ${ }^{16 a, d, 17}$ Using a concentric sphere capacitor model to describe the

(16) (a) Miles, D. T.; Murray, R. W. Anal. Chem. 2003, 75, 1251. (b) Schaaff, T. G.; Shafigullin, M. N.; Khoury, J. T.; Vezmar, I.; Whetten, R. L. J. Phys. Chem. B 2001, 105, 8785. (c) Hicks, J. F.; Miles, D. T.; Murray, R. W. J. Am. Chem. Soc. 2002, 124, 13322. (d) Quinn, B. M.; Liljeroth, P.; Ruiz, V.; Laaksonen, T.; Kontturi, K. J. Am. Chem. Soc. 2003, 125, 6644.

(17) (a) Chen, S.; Murray, R. W.; Feldberg, S. W. J. Phys. Chem. B 1998, 102 9898. (b) Miles, D. T.; Leopold, M. C.; Hicks, J. F.; Murray, R. W. J. Electroanal. Chem. 2003, 554-555, 87. 

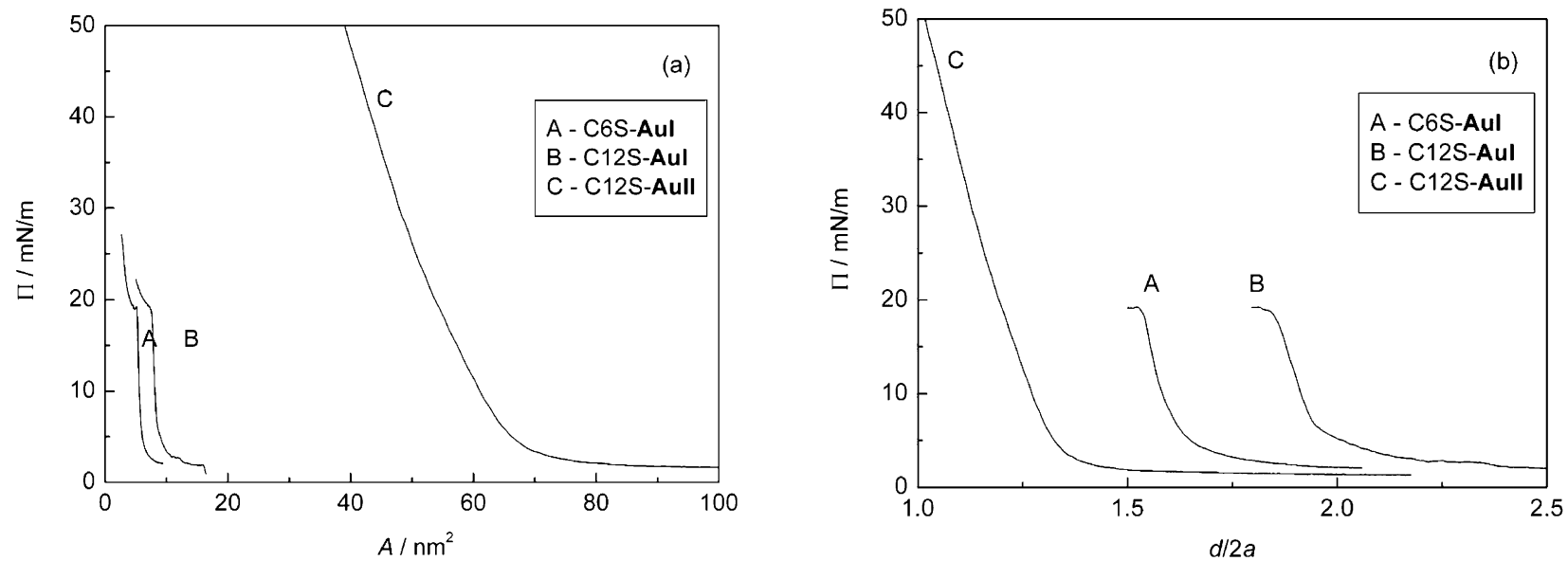

Figure 1. Isotherms of the nanoparticle films, the surface pressure, $\Pi$, as (a) a function of the area per particle, $A$, and (b) center-to-center distance between the particles, $d$, divided by the particle diameter, $2 a$. C6S-AuI (A, particle core diameter $1.6 \mathrm{~nm}$ ), C12S-AuI (B, particle core diameter $1.6 \mathrm{~nm}$ ), and C12SAuII (C, particle core diameter $6.6 \mathrm{~nm})$.

capacitance of the protecting thiol layer, we estimated the size of the gold core, yielding $1.6 \mathrm{~nm}$ as the particle diameter. ${ }^{17 \mathrm{a}, 18}$

To obtain uniform NCs of larger core size, the thermal annealing method reported by Shimizu et al. was used. ${ }^{19}$ Briefly, the as-prepared, crude dodecanethiol protected gold NCs were rotavapped to dryness without removal of the excess tetraoctylammonium salts, and the resulting black solid was heat-treated at $190{ }^{\circ} \mathrm{C}$ for $30 \mathrm{~min}$ in a furnace at a heating rate of $2{ }^{\circ} \mathrm{C} / \mathrm{min}$. After heat treatment, the product $(\mathrm{C} 12 \mathrm{~S}$ AuII) was cleaned by dissolution/precipitation cycles. Evolution of core size was confirmed by both the appearance of a surface plasmon in the UV spectra and TEM images of drop cast films on carbon-coated copper grids. ${ }^{19}$ The observed particle diameter for C12S-AuII was 6.6 $\pm 0.8 \mathrm{~nm}$ (Supporting Information). QDL charging is not resolvable at room temperature for particles with diameters greater than $2 \mathrm{~nm} .{ }^{17 \mathrm{a}}$

The absorption spectra of the chloroform solutions and transferred monolayer films were recorded using a HP 8452A diode array UVvis spectrophotometer. TEM measurements were conducted with a Philips CM200FEG, working at an accelerating voltage of $200 \mathrm{kV}$. The point-to-point resolution was $0.24 \mathrm{~nm}$. Images were recorded by the GATAN model 694 slow-scan $1024 \times 1024$ CCD camera. The particle size was measured directly from TEM images. Images were processed using FFT technique to enhance signal-to-noise ratio before measuring the interparticle separation.

Langmuir Deposition. The particles were spread from very dilute chloroform solutions (C6S-AuI $0.4 \mathrm{mg} / \mathrm{mL}, \mathrm{C} 12 \mathrm{~S}-\mathrm{AuI} 0.114 \mathrm{mg} / \mathrm{mL}$, C12S-AuII $0.22 \mathrm{mg} / \mathrm{mL}$ ) onto a pure water (milli-Q, Millipore) subphase in a KSV mini-trough (KSV instruments, Finland) thermostated at $20^{\circ} \mathrm{C}$. Initially, the resulting film was composed of voids and islands but formed a homogeneous film with time as previously reported. ${ }^{5 b, c}$ Thus, the film was allowed to equilibrate for typically $2 \mathrm{~h}$ prior to compression. The compression rate used throughout was $5 \mathrm{~mm} /$ $\mathrm{min}$. The NC monolayer was compressed to the desired surface pressure and held at that pressure to allow for relaxation of the monolayer $(90$ min). A silanized glass slide was brought down horizontally by hand to touch the monolayer and lifted carefully after a contact time of a few seconds. The area occupied per NC, $A$, was calculated on the basis of the trough area and the amount of particles spread. The fact that transferred monolayers redispersed in chloroform shows that irreversible aggregation of the metal cores had not occurred during compression.

Microscope slides were cut into ca. $1 \mathrm{~cm}^{2}$ squares and were cleaned in piranha solution (caution: piranha solution reacts violently with organics; it should be handled with extreme caution!). The surface was rendered hydrophobic by immersion in dimethyldichlorosilane (Fluka) solution for $30 \mathrm{~min}$, rinsed well with toluene to remove unreacted silane, and dried in the oven for $15 \mathrm{~min}$. Silanization of the surface was essential for successful transfer of the NC films.
For films of C12S-AuII, TEM images of the films transferred by the Langmuir-Schaefer method onto carbon coated grids at various surface pressures were also obtained. In the statistical determination of the interparticle distance, lattice defects were ignored.

SECM Measurements. SECM measurements were performed using a commercially available SECM instrument (CHI-900, CH-Instruments, $\mathrm{TX})$. A two-electrode arrangement with a silver wire as quasi-reference electrode and counter electrode was used. The working electrode used throughout was a $25 \mu \mathrm{m}$ diameter Pt ultramicroelectrode (UME). Diskshaped Pt SECM tips were prepared as previously described. ${ }^{20}$ The tips used had $R_{\mathrm{G}}=5$ (ratio of the overall tip radius, $r_{\mathrm{g}}$, to that of the platinum disk, $r_{\mathrm{e}}$ ) as determined from both optical micrographs and SECM approach curve experiments to insulating (PTFE) and conducting (Pt) substrates followed by fitting the results to the approximations provided by Amphlett and Denuault. ${ }^{21}$

A schematic of the experimental arrangement is given in Scheme 1. The experimental protocol employed to obtain approach curves at low redox mediator concentrations has been described in detail previously. ${ }_{15 \mathrm{~b}, \mathrm{c}}$ Ferrocene methanol (Sigma) and lithium chloride (concentration typically $0.1 \mathrm{~mol} / \mathrm{dm}^{3}$ ) were used as the redox mediator and base electrolyte, respectively, in all experiments.

\section{Results and Discussion}

The Langmuir isotherms for the three NC monolayers considered are given in Figure 1 in terms of both (a) area per $\mathrm{NC}$ and (b) the normalized interparticle separation $d / 2 a$, where $d$ is the center-to-center distance between adjacent NCs and $a$ is the core radius ( $d$ was calculated on the basis of the area per particle assuming hexagonal packing of NCs). The shape of the isotherms and the area per particle are consistent with previous reports. ${ }^{1 \mathrm{a}, 5 \mathrm{~b}, 8-10,22}$ From previous reports by Heath and coworkers, where an $\mathrm{I}-\mathrm{M}$ transition was noted for comparable silver NC monolayers, $d / 2 a$ should be less than 1.2 to have sufficiently strong exchange coupling between adjacent NCs to overcome the charging energy between them. ${ }^{1 \mathrm{a}, 6 \mathrm{a}}$ If this criterion is met, in the case of suitably monodisperse NCs, the

(18) Hicks, J. F.; Templeton, A. C.; Chen, S.; Sheran, K. M.; Jasti, R.; Murray, R. W.; Debord, J.; Schaaff, T. G.; Whetten, R. L. Anal. Chem. 1999, 71, 3703.

(19) Shimizu, T.; Teranishi, T.; Hasegawa, S.; Miyake, M. J. Phys. Chem. B 2003, 107, 2719

(20) Bard, A. J.; Fan, F. R. F.; Mirkin, M. V. In Electroanalytical Chemistry; Bard, A. J., Ed.; Marcel Dekker: New York, 1994; Vol. 18; p 243. 


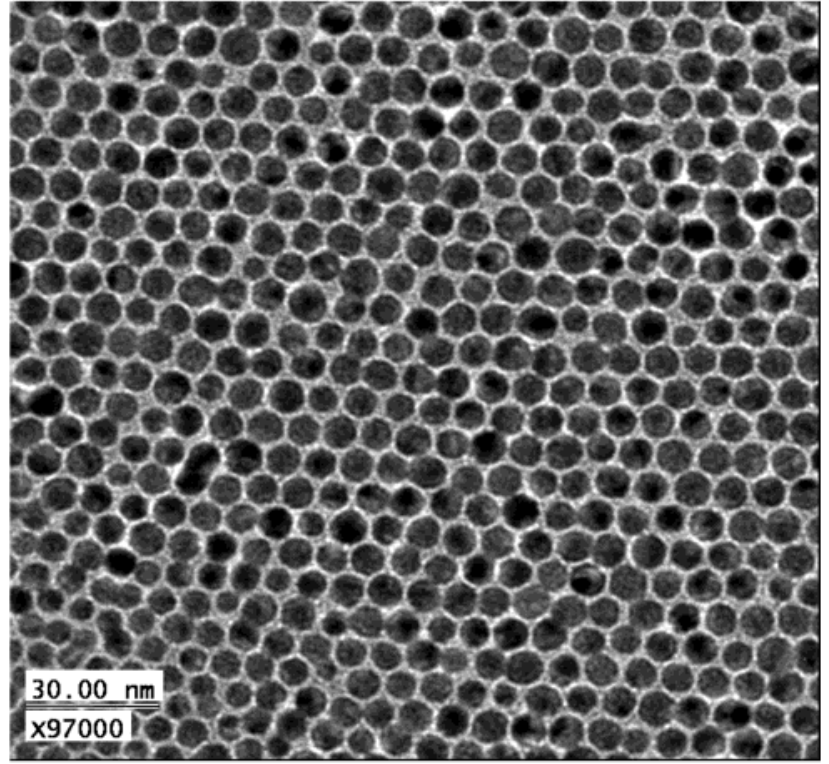

Figure 2. TEM image of C12S-AuII (core diameter $6.6 \mathrm{~nm}$ ) film transferred at a surface pressure of $20 \mathrm{mN} / \mathrm{m}$.

Table 1. Particle Sizes (2a), Deposition Surface Pressures $\left(\Pi_{\text {dep }}\right)$, Area Per Particle $(A)$, and Interparticle Separations, in Terms of Both Center-to-Center Distance $(d)$ normalized by the Particle Diameter and the Actual Core-to-Core Distance, for the Studied Monolayers

\begin{tabular}{lcrrrrrr}
\hline \multicolumn{1}{c}{ particles } & $\begin{array}{c}2 a \\
(\mathrm{~nm})\end{array}$ & $\begin{array}{c}\Pi_{\text {dep }} \\
(\mathrm{mN} / \mathrm{m})\end{array}$ & $\begin{array}{c}A \\
\left(\mathrm{~nm}^{2}\right)\end{array}$ & $\begin{array}{c}d / 2 a^{a} \\
(\mathrm{~nm})\end{array}$ & $\begin{array}{c}d-2 a^{a} \\
d / 2 a^{b}\end{array}$ & $\begin{array}{c}d-2 a^{b} \\
(\mathrm{~nm})\end{array}$ \\
\hline C6S-AuI & 1.6 & 15 & 5.4 & 1.55 & 0.9 & & \\
C12S-AuI & 1.6 & 15 & 7.8 & 1.88 & 1.4 & & \\
C12S-AuII & 6.6 & 3 & 71.5 & 1.38 & 2.5 & 1.23 & 1.5 \\
C12S-AuII & 6.6 & 10 & 61.0 & 1.27 & 1.8 & 1.20 & 1.3 \\
C12S-AuII & 6.6 & 15 & 57.4 & 1.23 & 1.5 & & \\
C12S-AuII & 6.6 & 20 & 53.7 & 1.19 & 1.3 & 1.20 & 1.3 \\
\hline
\end{tabular}

$a$ Obtained from the Langmuir experiments. ${ }^{b}$ Obtained from TEM.

monolayer behaves as a metal at room temperature. ${ }^{2 \mathrm{~b}, 4 \mathrm{~h}, 5 \mathrm{a}, \mathrm{b}, 14,22 \mathrm{a}}$ As can be seen from Figure 1b, for the $1.6 \mathrm{~nm}$ diameter NC cores, this is not fulfilled: the monolayer is in the collapsed state already at 1.8 for the C12S-AuI and at 1.4 for the C6SAuI. It should be realized, however, that while $d / 2 a$ is 1.23 and 1.88 for C12S-AuII and C12S-AuI, respectively, at a surface pressure of $15 \mathrm{mN} / \mathrm{m}$, the actual core edge-to-edge separation $(d-2 a)$ is actually less in the latter case $(1.40$ compared to $1.54 \mathrm{~nm}$ ). Thus, if the relevant parameter characterizing the electrical behavior of the monolayer is $d / 2 a$, then for the same thiol chain length, it is experimentally easier to attain significant coupling between the particles upon film compression by making the NC cores larger.

Monolayers were transferred using the Langmuir-Schaefer technique (horizontal deposition) at the desired surface pressure, both on silanized glass slides and carbon-coated TEM grids. A TEM image of a monolayer transferred at a surface pressure of $20 \mathrm{mN} / \mathrm{m}$ is shown in Figure 2, where the high quality hexagonal packing is evident. TEMs at a higher surface pressure $(50 \mathrm{mN} /$ m) show evidence of monolayer collapse and the resulting loss

(22) (a) Henrichs, S.; Collier, C. P.; Saykally, R. J.; Shen, Y. R.; Heath, J. R J. Am. Chem. Soc. 2000, 122, 4077. (b) Brust, M.; Stuhr-Hansen, N.; Nørgaard, K.; Christensen, J. B.; Nielsen, L. K.; Bjørnholm, T. Nano Lett. 2001, 1, 189. (c) Nørgaard, K.; Weygand, M. J.; Kjaer, K.; Brust, M.; Bjørnholm, T. Faraday Discuss. 2003, 125. of monolayer order (Supporting Information). The TEM images were analyzed to yield the interparticle distances, and the results are shown in the two rightmost columns of Table 1. This table also gives the area per particle and the corresponding centerto-center and core-to-core distances as obtained from the Langmuir isotherms.

Optical absorbance spectra of the transferred films are shown in Figure 3. C6S-AuI particles do not display a plasmon peak in the spectrum obtained with NCs dispersion in chloroform due to their small size $(<2 \mathrm{~nm})$. However, in a close-packed film, the dipole coupling between the particles causes a plasmon resonance to be visible. C12S-AuI behaves similarly to C6SAuI both in solution and monolayers. The larger particles, C12SAuII, display a clear plasmon resonance peak at ca. $520 \mathrm{~nm}$, which is significantly red-shifted to ca. $600 \mathrm{~nm}$ in the films. This shift is qualitatively well understood within the framework of effective medium theories, such as the Maxwell-Garnett (MG) model. ${ }^{23}$ It is the dielectric coupling between the particles that is responsible for the change in the complex dielectric permittivity of the films and, hence, the shift in the plasmon resonance. If the observed dependence of the shift on $d / 2 a$ is considered, it is considerably weaker than that predicted by MG model. The line shape is also changed upon compression of the film, with the peak width decreasing by ca. $20 \%$ going from $d / 2 a=1.4$ to 1.2 . Heath and co-workers attribute these effects, i.e., the weaker than expected red-shift of the plasmon resonance and the simultaneous line narrowing, to electronic coupling of the particles. ${ }^{23}$

Examples of experimental SECM approach curves, where the current, $I$, is scaled by the limiting current far from the substrate, $I_{\text {lim }}=4 n F D c^{\mathrm{b}} r_{\mathrm{e}}$ ( $n$ is the number of electrons transferred, $F$ the Faraday constant, $D$ and $c^{\mathrm{b}}$ are the diffusion coefficient and the bulk concentration of the solution redox couple, respectively), to transferred monolayers at defined surface pressures are given in Figure 4. The observed feedback response is a combination of the diffusion flux in the solution and the flux due to lateral electron transport in the film. As the diffusion flux is directly proportional to the concentration of the redox mediator in solution, to observe lateral transport, the concentration has to be reduced sufficiently. As the monolayers are transferred onto an insulating substrate, at high redox couple concentrations, negative feedback due to hindered diffusion to the SECM tip is observed. As the concentration is reduced, the relative contribution of mediator regeneration due to lateral electron transport in the film increases. At a given concentration, dependent on the monolayer conductivity, deviation from purely negative feedback will be observed. Thus, from the concentration dependence of the observed feedback response, the lateral conductivity of the monolayer can be quantified using the model outlined below. The concentration dependence of the approach curves shown in Figure 4 reveals that deviation is apparent already at high concentrations $(1.07 \mathrm{mM})$ for C12S-AuII monolayers but only at extremely low concentrations $(1.6 \mu \mathrm{M})$ for that of C12S-AuI. The turn-on concentration for the other $\mathrm{NC}$ monolayer considered (C6S-AuI) was of the same order of magnitude as that for C12s-AuI (Supporting Information). In

(23) Shiang, J. J.; Heath, J. R.; Collier, C. P.; Saykally, R. J. J. Phys. Chem. B 1998, 102,3425 . 

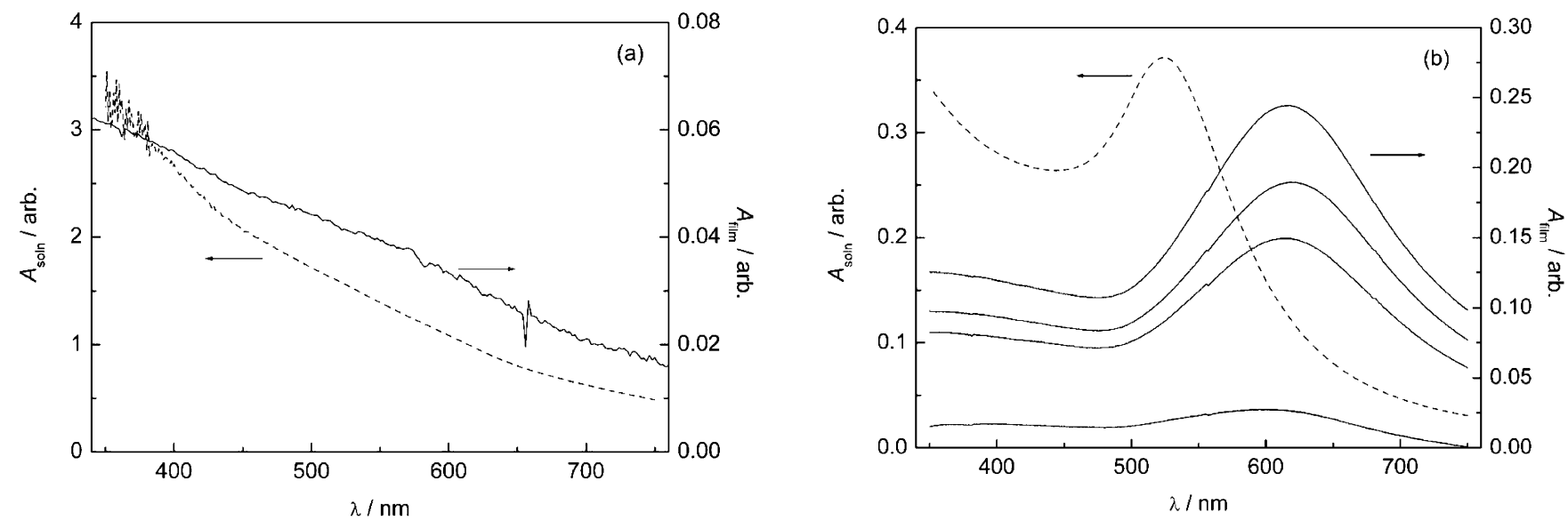

Figure 3. UV-vis absorbance spectra for the transferred films of (a) C6S-AuI (deposition pressure $15 \mathrm{mN} / \mathrm{m}$ ) and (b) C12S-AuII (deposition pressure from bottom to top, $3,10,15$, and $20 \mathrm{mN} / \mathrm{m}$ ). The absorbance in solution (chloroform) is shown as a dashed line.
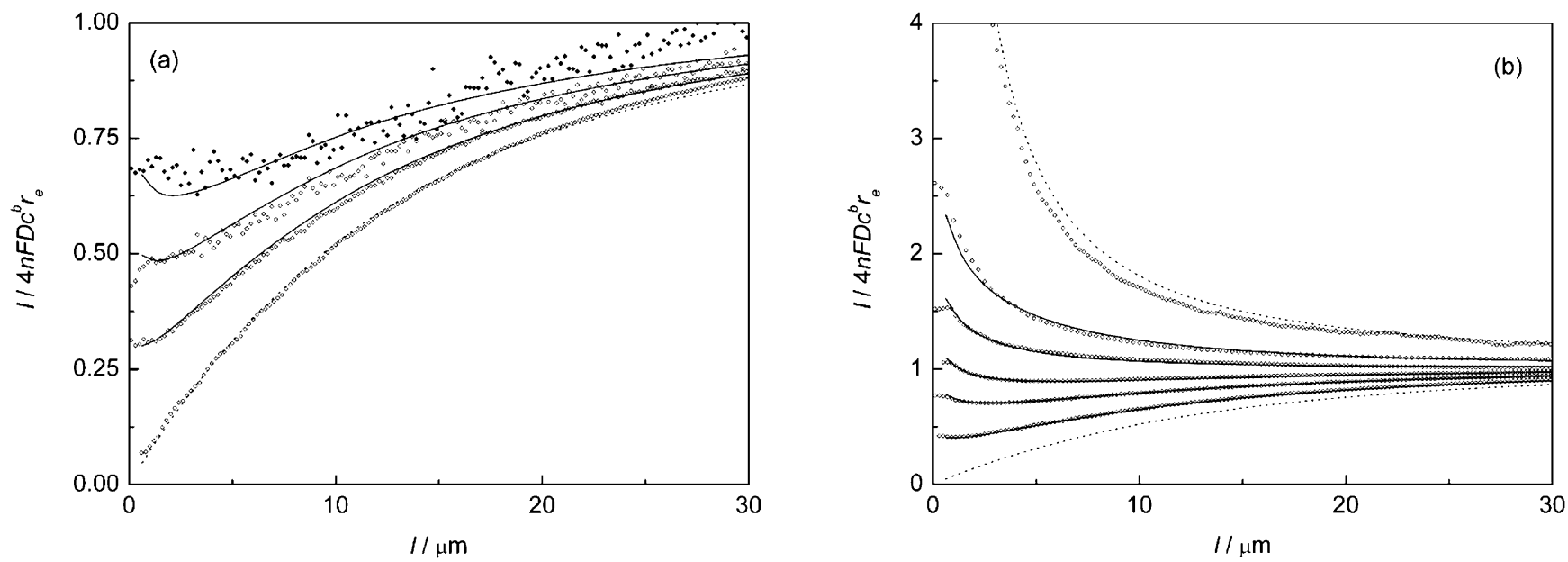

Figure 4. Examples of experimental approach curves (dots), where the normalized tip current is plotted as a function of tip-monolayer distance, $l$, and the corresponding fits to the theory (solid lines). (a) C12-AuI (core diameter $1.6 \mathrm{~nm}$ ) transferred at a surface pressure of $15 \mathrm{mN} / \mathrm{m}$, concentrations of $57,1.6$, 0.5, $0.2 \mu \mathrm{M}$ (from bottom to top, lowest concentration in closed symbols). (b) C12S-AuII (core diameter $6.6 \mathrm{~nm}$ ) transferred at a surface pressure of 10 $\mathrm{mN} / \mathrm{m}$, concentrations of $1070,450,280,170,60$, and $1.3 \mu \mathrm{M}$ (from bottom to top). The lower and upper dashed lines are the theoretical responses for negative and positive feedback, respectively.

fact, for the C12S-AuII monolayer, we were unable to observe a pure negative feedback response due to solubility limitation of ferrocene methanol in water.

The experimental approach curves can be fitted to the theory outlined below, and the corresponding fits are also given in Figure 4 (solid lines). The model assumes that the monolayer exhibits ohmic behavior, i.e., the conduction current is directly proportional to the local electrochemical potential gradient. This assumption is valid in the absence of Coulomb blockade and for low potential gradients (i.e., driving force). Despite the large charging energy of the smaller NCs (core diameter $1.6 \mathrm{~nm}$, charging energy of ca. $250 \mathrm{meV}$ in organic solvents), linear current-voltage relationships have been observed in the literature. ${ }^{4 a, d, 24}$ Ohmic conduction in a polyaniline monolayer has been considered in the literature. ${ }^{25}$ However, this is the first study where the coupling between the electron transport in the monolayer and diffusion in solution is treated explicitly. The solution of the electrochemical mass-transport problem involves the following transport equation on the substrate coupled with

(24) Zamborini, F. P.; Leopold, M. C.; Hicks, J. F.; Kulesza, P. J.; Malik, M. A.; Murray, R. W. J. Am. Chem. Soc. 2002, 124, 8958.

(25) Zhang, J.; Barker, A. L.; Mandler, D.; Unwin, P. R. J. Am. Chem. Soc. 2003, 125,9312 . the diffusion equation in solution (Supporting Information)

$$
\frac{\partial^{2} \tilde{\mu}}{\partial R^{2}}+\frac{1}{R} \frac{\partial \tilde{\mu}}{\partial R}-\frac{K^{0}}{\Sigma}\left((1-C) e^{\tilde{\mu} / 2}-C e^{-\tilde{\mu} / 2}\right)=0
$$

where $\tilde{\mu}=\left(\mu-\mu^{0}\right) / k T$ is the dimensionless electrochemical potential of the electrons in the $\mathrm{NC}$ monolayer, $K^{0}$ is the dimensionless standard rate constant of the electron-transfer reaction between the solution redox couple and the nanoparticle film, $C$ is the dimensionless concentration of the solution redox couple at the substrate, and $\Sigma$ is the dimensionless conductivity in the film given by the following equation

$$
\Sigma=\frac{\sigma k T \Delta z}{e^{2} r_{e} D c^{\mathrm{b}} N_{\mathrm{A}}}
$$

where $\sigma$ is conductivity, $k$ is the Boltzmann constant, $T$ is the absolute temperature, $\Delta z$ is the thickness of the film (taken as the diameter of the particles), $e$ is the elementary charge, and $N_{\mathrm{A}}$ is the Avogadro constant. Equation 1 has a boundary condition $\tilde{\mu}(R \rightarrow \infty)=\tilde{\mu}_{\mathrm{eq}}$, which implies that the electrochemical potential of the electrons in the NCs attains an equilibrium value far from SECM tip (Supporting Information). The kinetics 

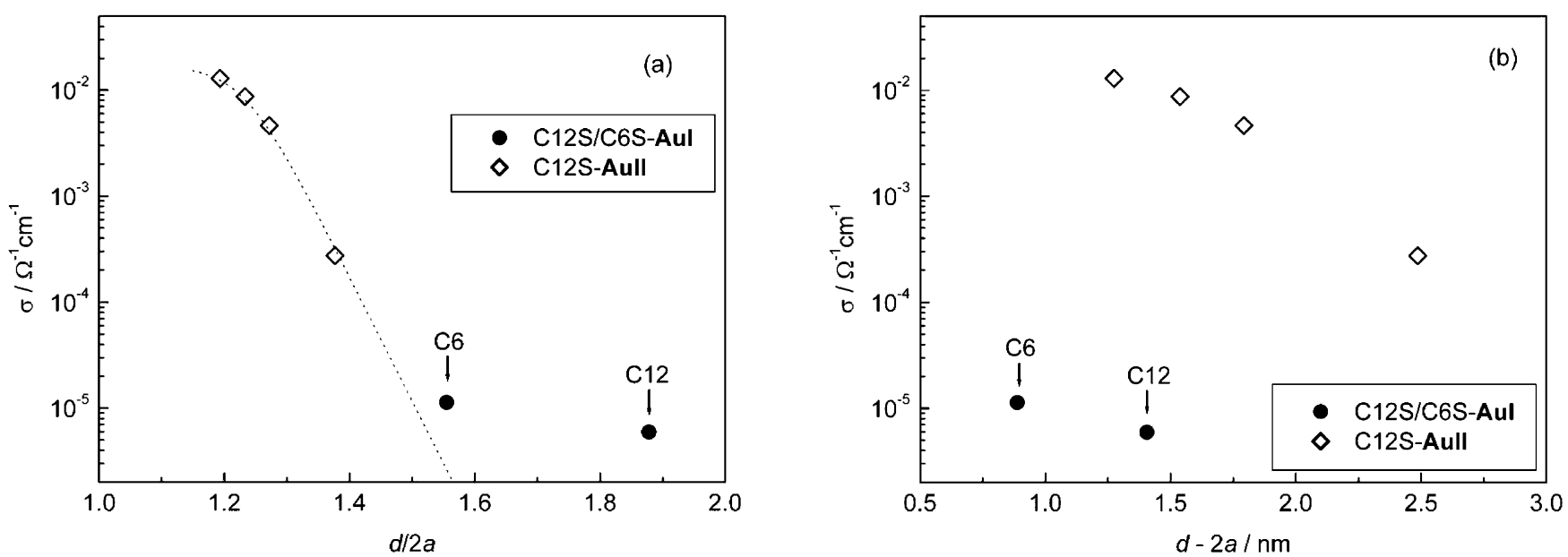

Figure 5. Conductivity extracted from the fitting of the experimental approach curves to the theoretical predictions for the nanoparticle films as a function of (a) the center-to-center distance scaled by the particle diameter, $d / 2 a$, and (b) the core-to-core distance, $d-2 a$. The dotted line in (a) is a best fit to the function $\sigma=\left(\sigma_{0} / 2\right)\left(1+\tanh \left[\left(d_{0}-d\right) / 4 a L\right)\right]$ (see text for details).

of electron transfer between the solution redox couple and the nanoparticle film enter through $K^{0}$. If kinetics of electron transfer played a role, the current would not increase at small SECM tip-monolayer separation. In addition, at low redox mediator concentration, pure positive feedback would not be observed. Experimentally, such kinetic limitations were not observed when ferrocene methanol was used as the redox mediator, and therefore, the approach curves were fitted with a large value of $K^{0}$ (=100). The value of the equilibrium electrochemical potential of the film has only a minor effect on the approach curves (Supporting Information). In conclusion, it should be borne in mind that, despite the apparent complexity of the model, in the end the fitting only involves one parameter, the film conductivity. Families of approach curves were generated where this parameter was varied. These were, in turn, used to fit the experimental response, and as can be seen from Figure 4, the correspondence between theory and experiment is excellent.

From the parameters used to obtain the best fit to the experimental approach curves, monolayer conductivity as a function of interparticle separation (determined by the surface pressure at which the film was transferred) was obtained. These values are plotted in Figure 5 as a function of both (a) the normalized center-to-center distance $d / 2 a$ and (b) the actual coreto-core separation $d-2 a$. It can be clearly seen that the $\mathrm{C} 12 \mathrm{~S}$ AuII monolayers are orders of magnitude more conductive compared with AuI particle films and that the conductivity increases exponentially as the interparticle separation is decreased. The conductivity values for the AuI NC films are comparable to previous reports. ${ }^{10,15 b, c}$ Also from the figure, it can be seen that for identical surface pressures, the conductivity of the C6S-AuI monolayer is twice that of the C12S-AuI monolayer. Figure 5 strongly supports the use of $d / 2 a$ as the relevant parameter in characterizing interparticle coupling in NC monolayers.

It could be argued that the observed increase is due to aggregation or the formation of multilayers. However, TEM images of the C12S-AuII films at various compressions show highly ordered monolayer arrays and that collapse is only apparent at $50 \mathrm{mN} / \mathrm{m}$. Moreover, all transferred films redispersed in chloroform post SECM measurements giving deep red solutions identical to the spreading solution, indicating that irreversible aggregation has not occurred. Thus, it can be stated that the increase in conductivity is due to the increased coupling between adjacent metal cores as the separation between them is decreased. The observed saturation of the conductivity at low $d / 2 a$ may be the room-temperature signature of an I-M transition for $\mathrm{Au}$ NCs observed previously for $\mathrm{Ag} \mathrm{NCs,} \mathrm{vide}$ infra. To truly classify the state as metallic, however, the temperature dependence of the conductivity should be addressed. This will be considered in a future publication.

In the work carried out by Heath et al., it was shown that the second harmonic generation response and the frequency dependent dielectric constant of Ag NC monolayers could be reproduced if the coupling, $\beta$, between the particles was assumed to be of the following functional form $\beta=\left(\beta_{0} / 2\right)\left(1+\tanh \left[\left(d_{0}\right.\right.\right.$ $-d) / 4 a L])$, where values of $d_{0} / 2 a=1.2$ and $1 / 2 L=5.5$ were obtained from fitting to experimental results. ${ }^{5 \mathrm{c}, 26}$ The parameter $d_{0} / 2 a$ determines the position of the transition from weak to strong coupling, and $1 / 2 L$ characterizes the sharpness of the transition. The dotted line in Figure 5a shows similar function, $\sigma=\left(\sigma_{0} / 2\right)\left(1+\tanh \left[\left(d_{0}-d\right) / 4 a L\right]\right)$, fitted to the conductivity data obtained from the SECM measurements for the $6.6 \mathrm{~nm}$ diameter NCs. The parameter values obtained are $d_{0} / 2 a=1.23$ and $1 / 2 L=13.5$, indicating that the transition we observe occurs at very similar interparticle separations noted previously for $\mathrm{Ag}$ NC monolayers. The transition observed here is sharper than that reported for $\mathrm{Ag} \mathrm{NC}$ monolayers, as indicated by the values for $1 / 2 L$ of 13.5 vs 5.5 . The range of $d / 2 a$ over which the transition occurs may be influenced by the method used to detect it: electrical instead of optical measurements. Polydispersity of the NCs might also affect sharpness of the transition as this reflects the extent of carrier delocalization in the monolayer. It is of interest to note that the saturation value of the conductivity (obtained by an extrapolation) is $\sigma_{0} \approx 0.02 \Omega^{-1} \mathrm{~cm}^{-1}$, which is very similar to the values obtained for $\mathrm{Ag} \mathrm{NC}$ monolayers compressed through the I-M transition. ${ }^{11}$

In summary, we have used an advanced electrochemical technique to probe the conductivity of gold NC monolayers. The results indicate that monolayers deposited at the highest compression $(d / 2 a<1.2$, deposition pressure $20 \mathrm{mN} / \mathrm{m})$, exhibit

(26) Remacle, F.; Levine, R. D. J. Am. Chem. Soc. 2000, 122, 4084. 
strong electronic coupling between the particles. This is taken as an indication of an insulator to metal transition in the monolayer and, as such, is the first report of this type of transition for $\mathrm{Au} \mathrm{NC}$ monolayers. The I-M transition had previously been reported exclusively for Ag monolayers, and the observation of a comparable transition here supports the assumption that this is a general feature of metal NC monolayers.

Acknowledgment. This work was funded by the National Technology Agency, Finland, the Academy of Finland, and the EU under the Sixth Framework Program "SUSANA" (EU TMR network "Supramolecular Self-Assembly of Interfacial Nanostructures", Contract No. HPRN-CT-2002-00185). We are grateful to James Heath and Kristen Beverly for making the values of conductivity for Ag NC monolayers available.

Supporting Information Available: Additional experimental results (particle size distribution for C12S-AuII, TEM image of a collapsed monolayer, SECM approach curves) and theoretical details for the SECM model (PDF). This material is available free of charge via the Internet at http://pubs.acs.org.

JA0493188 